\title{
Toward better risk stratification of asymptomatic Brugada Syndrome patients?
}

\author{
Najla Beydoun ${ }^{1}$, Charbel Gharios ${ }^{2}$, and Marwan Refaat ${ }^{3}$ \\ ${ }^{1}$ Tufts Medical Center \\ ${ }^{2}$ Massachusetts General Hospital \\ ${ }^{3}$ American University of Beirut Medical Center
}

September 29, 2021

\section{Toward Better Risk Stratification of Asymptomatic Brugada Syndrome Patients?}

Najla Beydoun $\mathrm{MD}^{1}$, Charbel Gharios $\mathrm{MD}^{2}$, Marwan M. Refaat $\mathrm{MD}^{3}$

${ }^{1}$ Department of Anesthesiology and Perioperative Medicine, Tufts Medical Center, Boston, MA

${ }^{2}$ Cardiovascular Imaging Research Center, Massachusetts General Hospital and Harvard Medical School, Boston, MA

${ }^{3}$ Department of Internal Medicine, Division of Cardiology, and Department of Biochemistry and Molecular Genetics, American University of Beirut Faculty of Medicine and Medical Center, Beirut, Lebanon

Short Title: Risk Stratification in Asymptomatic BrS

Word Counts: 602

Conflict of Interests

The authors declare no conflicts of interest

\section{Funding}

None

\section{Keywords}

Cardiac arrhythmias, Brugada Syndrome, risk stratification, electroanatominal mapping, cardiovascular diseases, heart diseases

\section{Hosted file}

image1.emf available at https://authorea.com/users/438593/articles/539755-toward-betterrisk-stratification-of-asymptomatic-brugada-syndrome-patients

The prognostic value of invasive electrophysiologic (EP) testing in asymptomatic Brugada Syndrome (BrS) patients remains controversial. Even though EP testing was recommended for the prognostication of asymptomatic BrS patients in the 2005 consensus statement on $\mathrm{BrS}^{1}$, more recent guidelines have decreased the emphasis on invasive testing for risk stratification. The $2013 \mathrm{HRS}$ /EHRA/APHRS consensus statement notes that there is no consensus on the prognostic value of EP testing, and the more recent $2017 \mathrm{AHA} / \mathrm{ACC} / \mathrm{HRS}$ guidelines mention that invasive testing may be considered in risk stratification of asymptomatic patients. Specifically, the prognostic role of programmed ventricular stimulation (PVS) for evaluation of ventricular arrhythmia inducibility has shown inconsistent results. ${ }^{2,} 3$ However, EP testing with myocardial substrate 
mapping has recently shown interesting results for the assessment of arrhythmia inducibility. In 191 patients with BrS who underwent EP testing, arrhythmogenic substrate size was an independent predictor of inducibility, and substrate ablation prevented ventricular arrhythmia re-inducibility. ${ }^{4}$

In this issue of the Journal of Cardiovascular Electrophysiology, Letsas et. al suggest a potential role for high-density electroanatomic mapping (HDEAM) in the risk stratification of BrS patients. To this end, they characterize the differences in endocardial abnormalities in the right ventricular outflow tract (RVOT) among high-risk and asymptomatic BrS patients. Given that epicardial RVOT abnormalities are believed to be the arrhythmogenic substrate in $\mathrm{BrS}^{5,6}$, and given that endocardial unipolar HDEAM helps in the localization of epicardial abnormalities ${ }^{7,8}$, the authors aimed to evaluate the clinical significance of looking at abnormal endocardial substrate.

In their retrospective analysis of prospectively collected data from 54 patients with BrS, fourteen symptomatic patients with aborted sudden cardiac death (SDC) underwent combined endocardial-epicardial HDEAM, while 40 asymptomatic patients underwent endocardial HDEAM. Comprehensive clinical evaluation and coronary angiography were performed to rule out structural heart disease and coronary artery disease, respectively. Unipolar and bipolar signals were recorded and the duration, amplitude, presence of multiple components and relation to surface QRS were analyzed. Abnormal endocardial findings were defined as low-voltage areas (LVAs) $>1 \mathrm{~cm}^{2}$ with unipolar signals $<5.3 \mathrm{mV}$. This value was based upon its $92.4 \%$ specificity and $86.3 \%$ sensitivity for the identification of epicardial bipolar LVAs $<1 \mathrm{mV}$. Moreover, all patients underwent programmed ventricular stimulation (PVS) from the RV apex and/or RVOT sites. In all cases, the endocardial unipolar LVAs were found to be wider than endocardial bipolar LVAs, and the abnormalities were mostly detected on the anterior wall of the RVOT. Interestingly, endocardial unipolar LVAs were found colocalize with epicardial bipolar LVAs. Importantly, symptomatic patients displayed wider endocardial unipolar and bipolar LVAs in comparison to asymptomatic ones. Based on receiver operatic characteristic (ROC) analysis, the presence of unipolar LVAs $>14.5 \mathrm{~cm}^{2}$ as well as that of bipolar LVAs $>3.68$ $\mathrm{cm}^{2}$ significantly discriminated symptomatic from asymptomatic individuals. Furthermore, all symptomatic patients and one fourth of the asymptomatic patients were inducible during PVS. Notably, wider areas of abnormal unipolar and bipolar signals were found in inducible patients compared to non-inducible ones.

These findings demonstrate that the size of endocardial abnormalities is associated with VF inducibility. In fact, unipolar LVAs $>13.5 \mathrm{~cm}^{2}$ and bipolar LVAs $>2.97 \mathrm{~cm}^{2}$ significantly predicted VF inducibility. Finally, all inducible patients with positive PVS received an implantable cardioverter defibrillator (ICD).

The study is limited by the small number of high-risk patients and the differences in catheter types and methods used to characterize the abnormal substrates, given the retrospective nature of the study. These results show different endocardial findings during HDEAM in high-risk BrS patients compared to asymptomatic patients. Whether these findings can be leveraged to better risk stratify asymptomatic BrS patients need to be investigated in future larger prospective cohorts.

\section{References}

1. Antzelevitch C, Brugada P, Borggrefe M, et al. Brugada syndrome: report of the second consensus conference: endorsed by the Heart Rhythm Society and the European Heart Rhythm Association. Circulation . Feb 8 2005;111(5):659-70. doi:10.1161/01.Cir.0000152479.54298.51

2. Sroubek J, Probst V, Mazzanti A, et al. Programmed Ventricular Stimulation for Risk Stratification in the Brugada Syndrome: A Pooled Analysis. Circulation . Feb 16 2016;133(7):622-30. doi:10.1161/circulationaha.115.017885

3. Kusumoto FM, Bailey KR, Chaouki AS, et al. Systematic review for the 2017 AHA/ACC/HRS guideline for management of patients with ventricular arrhythmias and the prevention of sudden cardiac death: A Report of the American College of Cardiology/American Heart Association Task Force on Clinical Practice Guidelines and the Heart Rhythm Society.Heart Rhythm . Oct 2018;15(10):e253-e274. doi:10.1016/j.hrthm.2017.10.037 
4. Pappone C, Ciconte G, Manguso F, et al. Assessing the Malignant Ventricular Arrhythmic Substrate in Patients With Brugada Syndrome.J Am Coll Cardiol . Apr 17 2018;71(15):1631-1646. doi:10.1016/j.jacc.2018.02.022

5. Frustaci A, Priori SG, Pieroni M, et al. Cardiac histological substrate in patients with clinical phenotype of Brugada syndrome.Circulation . Dec 13 2005;112(24):3680-7. doi:10.1161/circulationaha.105.520999

6. Pieroni M, Notarstefano P, Oliva A, et al. Electroanatomic and Pathologic Right Ventricular Outflow Tract Abnormalities in Patients With Brugada Syndrome. J Am Coll Cardiol . Dec 4 2018;72(22):2747-2757. doi:10.1016/j.jacc.2018.09.037

7. Polin GM, Haqqani H, Tzou W, et al. Endocardial unipolar voltage mapping to identify epicardial substrate in arrhythmogenic right ventricular cardiomyopathy/dysplasia. Heart Rhythm. Jan 2011;8(1):7683. doi:10.1016/j.hrthm.2010.09.088

8. Venlet J, Piers SRD, Kapel GFL, et al. Unipolar Endocardial Voltage Mapping in the Right Ventricle: Optimal Cutoff Values Correcting for Computed Tomography-Derived Epicardial Fat Thickness and Their Clinical Value for Substrate Delineation. Circ Arrhythm Electrophysiol . Aug 2017;10(8)doi:10.1161/circep.117.005175 